\title{
OPTICAL CHARACTERISTICS OF CU-NANOCLUSTER LAYERS ASSEMBLED BY ION IMPLANTATION
}

\author{
R. F. HAGLUND Jr.(1), R. H. MAGRUDER IU(2), L. YANG(1), \\ J. E. WITTIG(2) and R.'A.'ZUHR(3) \\ (1)Department of Physics and Astronomy and \\ (2)Department of Materials Science and Engineering \\ Vanderbilt University, Nashville, TN 37235 U.S.A. \\ CONF-911033--4 \\ (3) Solid-State Physics Division, Oak Ridge National Laboratory \\ Oak Ridge, TN 37831 U.S.A.
}

\begin{abstract}
We have generated cluster layers in solid insulating substrates by implanting $\mathrm{Cu}$ ions into fused silica, creating thin layers $(-150 \mathrm{~nm})$ of nanoclusters over a diameter of order $2 \mathrm{~cm}$. Transmission electron microscopy shows that the size and size distribution can be controlled by the parameters of the ion implantation. We report measurements of the optical properties and nonlinear index of refraction on these unusual solid-phase cluster materials as a function of total implanted-ion dose.
\end{abstract}

\section{Introduction and Motivation}

Metallic clusters of nanometer dimensions have many desirable characteristics as optical materials: ultrafast switching times, high resistance to bulk and surface laser damage, and large third-order optical nonlinearities. ${ }^{1}$ Recently we have made nanometer-size copper clusters in dense, solid-phase layers by implanting $\mathrm{Cu}$ ions in glass substrates. ${ }^{2}$ Apart from the known nonlinear optical behavior of metal nanoclusters, we are motivated by the fact that ion implantation is already a standard technology for modifying the properties of semiconductors and forming optical waveguides in dielectric materials.

In this paper, we describe the creation of dense layers of nanometer-size clusters by ion implantation, and show that total ion dose significantly affects the size and size distribution of nanoclusters created by $\mathrm{Cu}$-ion implantation in fused silica. We describe the optical properties of these cluster-assembled layers, including absorption, index of refraction, and nonlinear index, and consider briefly the physical origin of these optical properties.

\section{Cluster Preparation and Characterization}

Ion implantation is a well-established technique for creating materials which cannot be generated by ordinary glass-forming processes which are constrained both by chemistry and by the thermodynamics of the glass transition.

As substrate materials we used optical quality silica (Spectrosil®) discs, $1 \mathrm{~mm}$ thick $\mathrm{x}$ $20 \mathrm{~mm}$ diameter. The nominal cation impurity content of the Spectrosil@ is less than 5 ppm by weight; the principal anion impurities are $\mathrm{Cl}(80 \mathrm{ppii})$ and $\mathrm{OH}(200 \mathrm{ppm})$. The disks were implanted in a vacuum of $10^{-7}$ Torr with $160 \mathrm{keV}$ singly-charged positive ions of $\mathrm{Cu}$ to doses of $6 \cdot 10^{16}$ (on both faces of the disc) and $12 \cdot 10^{16} \mathrm{ions} \cdot \mathrm{cm}^{-2}$ (on a single side). The ion beam was electrostatically rastered to provide uniformity of implantation, and the current density was set at $10 \mu \mathrm{A} \cdot \mathrm{cm}^{-2}$, holding the "global" temperature of the

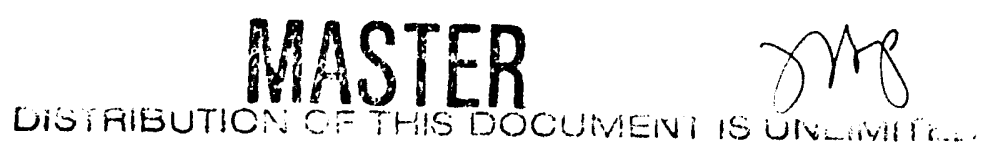

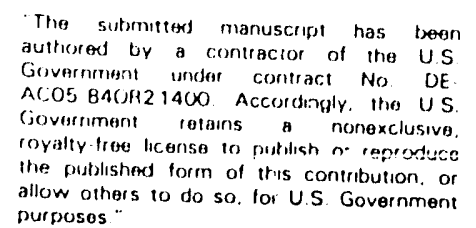


substrate below $50^{\circ} \mathrm{C}$. However, this does not preclude the possibility of strong localized heating in the implanted layer. Since these doses are above saturation, compaction effects due to radiation damage were approximately the same for both samples.

The depth distribution of the implanted $\mathrm{Cu}$ was measured by $\mathrm{He}^{+}$ion backscattering The total dose calculated from the backscattering measurements was typically $90 \%$ of the integrated current measured during implantation, indicating minimal sample charging. The volume concentration of the $\mathrm{Cu}$-layer at peak density is of order 0.1 . The backscattering profiles of the implanted layers are bimodal: the larger of the two peaks is twice the size of the smaller, and appears $85 \mathrm{~nm}$ below the surface, while the smaller peak is at a distance of $170 \mathrm{~nm}$ from the surface; the implanted layer was some $150 \mathrm{~nm}$ thick FWHM.

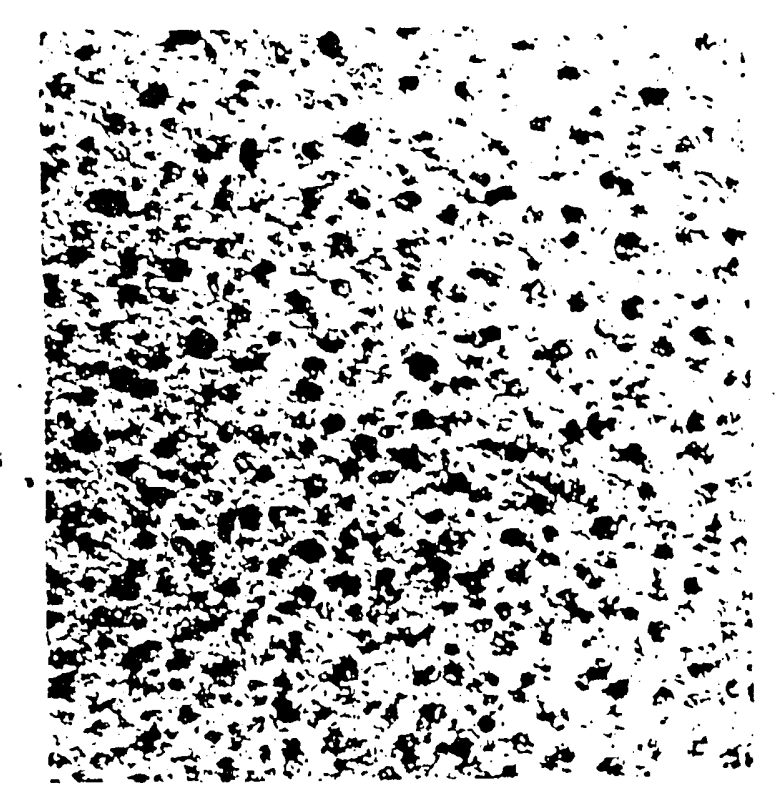

Figure 1. TEM micrograph of the layer implanted to a dose of $6 \cdot 10^{16}$ ions $\cdot \mathrm{cm}$.

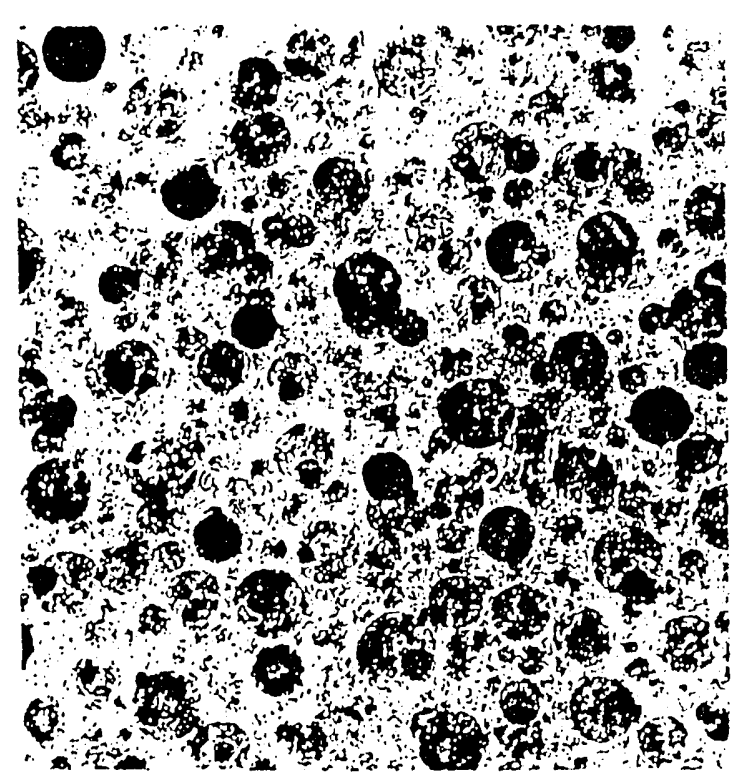

Figure 2. TEM micrograph of the layer implanted to a dose of $12 \cdot 10^{16}$ ions $\cdot \mathrm{cm}$.

The size and size distribution of the $\mathrm{Cu}$ nanoclusters were characterized by transmission electron microscopy (TEM). Samples for TEM were prepared by cutting a 3-mm-diameter disc from the implanted wafers with a slurry drill, mechanically grinding the disc from the back side to a thicknesss of about $150 \mu \mathrm{m}$, and dimple grinding to approximately $50 \mu \mathrm{m}$ from the implanted surface. Electron transparency was achieved by backthinning with an 5 $\mathrm{keV}$ Ar ion gun while cryogenically cooling the samples to minimize ion damage. The samples were then examined in a Philips CM20/T scanning transmission electron microscope operating at $200 \mathrm{kV}$. The high dose samples were stable in the microscope; the lower-dose samples, however, required carbon coating to eliminate charging effects.

Standard bright field (BF) imaging techniques were used to characterize the implanted layer. The BF images in Figures 1 and 2 show plane views of the ion-implanted layer. TEM scans made while tilting the sample revealed that the round particles embedded in the silica matrix are spherical in shape with random crystallographic orientation. An electron diffraction pattern taken from a selected area within the displayed region exhibited the polycrystalline ring pattern characteristic of an fcc metallic copper structure, superimposed on the diffuse diffracted intensity from the amorphous silıca matrix. 
An area of each micrograph containing approximately 200 particles was selected as the basis for measuring particle size distribution. Individual particle diameters were measured using a digital vernier caliper and tabulated; particles touching the boundary of the selected area were weighted by a factor 0.5. By measuring the boundary surface and making use of both the photographic and TEM magnifications, a surface area was calculated. It was assumed that the depth of the analyzed region was equal to the FWHM depth of the implanted layer as measured by the ion backscattering profile, about $150 \mathrm{~nm}$. This permits extraction of a volumetric density of the clusters. The density extracted by this means is in good agreement with the backscattering density for the $12.10^{16}$ ions $\mathrm{cm}^{-2}$ sample. For the $6 \cdot 10^{16}$ ions $\cdot \mathrm{cm}^{-2}$ sample, the correlation is not as good, suggesting that a significant fraction of the nanoclusters may have diameters below the resolution limit of the TEM.

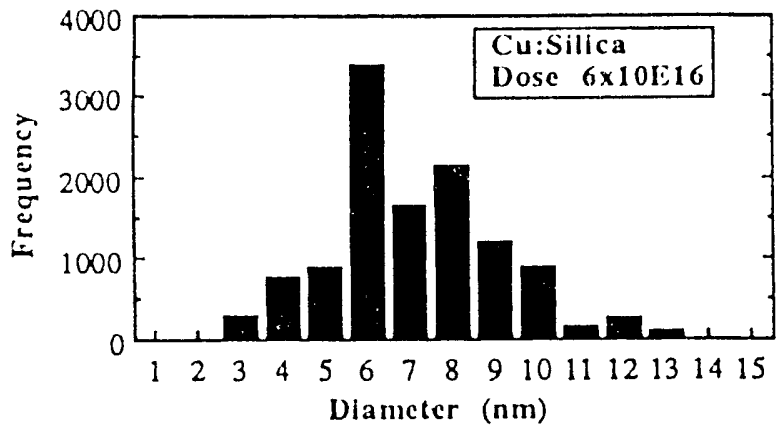

Figure 3 Distribution of cluster sizes in Cu:silica implanted to $6 \cdot 10^{16}$ ions $\cdot \mathrm{cm}$.

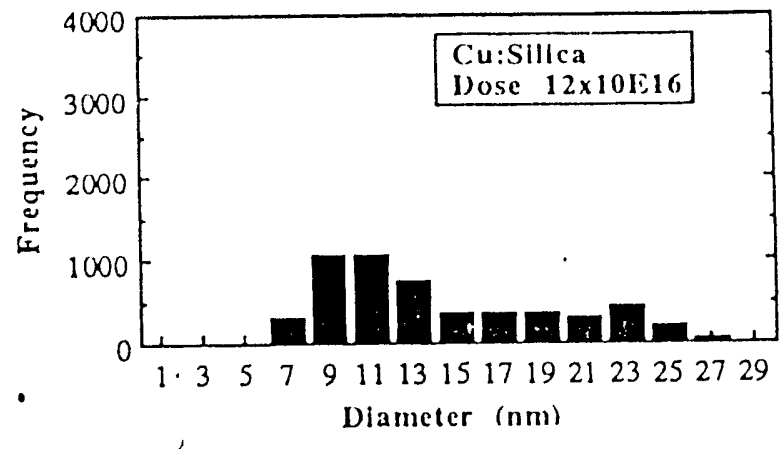

Figure 4 Distribution of cluster sizes in Cu:silica implanted to $12 \cdot 10^{16}$ ions $\cdot \mathrm{cm}$.

\section{Linear and Nonlinear Optical Properties of the Cluster Layer}

The TEM measurements provide important clarification for a variety of optical properties of cluster-assembled layers, including measurements of linear absorption, linear index of refraction, and nonlinear index of refraction.

Optical absorption spectra were measured in a Cary dual-beam spectrophotometer, using an unimplanted sample as a reference. The optical absorption spectra in Figure 5 show a peak at $2.2 \mathrm{eV}$ whose amplitude is dose dependent. The absorption in the 2.4 to $4.0 \mathrm{eV}$ region increases with dose. In the $4 \mathrm{eV}$ region the absorption per implanted ion is the same for both samples, while above $4.5 \mathrm{eV}$, the absorption per ion is again higher for the higherdose sample. We attribute the larger absorption in the 2.4 to $4 \mathrm{eV}$ region to the broader distribution of particle sizes in the higher-dose sample, as evident in the histogram of Figure 4. The peak at $2.2 \mathrm{eV}$ may be due to the bulk surface plasmon resonance peak.

Ellipsometric measurements were carried out on $\mathrm{Cu}$-implanted silica at a wavelength of $633 \mathrm{~nm}$ using a manual ellipsometer. Samples implanted with $\mathrm{Cu}$ and several transitionmetal elements from the same row of the periodic table over a wide range of doses were examined to determine their complex refractive indices $n=n_{0}+i k$. The data showed a sharp rise in index of refraction with increasing dose for the $\mathrm{Cu}$-implanted silica, as shown in Figure 6.3 In this region of the spectrum, the complex refractive index increases due to optical excitation of the surface plasmon resonance of the Cu clusters. ${ }^{4}$ Under these conditions, the imaginary part $k$ of the refractive index increases rapidly and largely determines 
the index. Incidentally, our recent infrared reflectance measurements ${ }^{5}$ show a red-shift in the location of the Si-O stretching mode peak with increasing ion dose. This behavior is consistent with breaking and reforming of the $\mathrm{Si}-\mathrm{O}$ bonds as the nanoclusters grow in size.

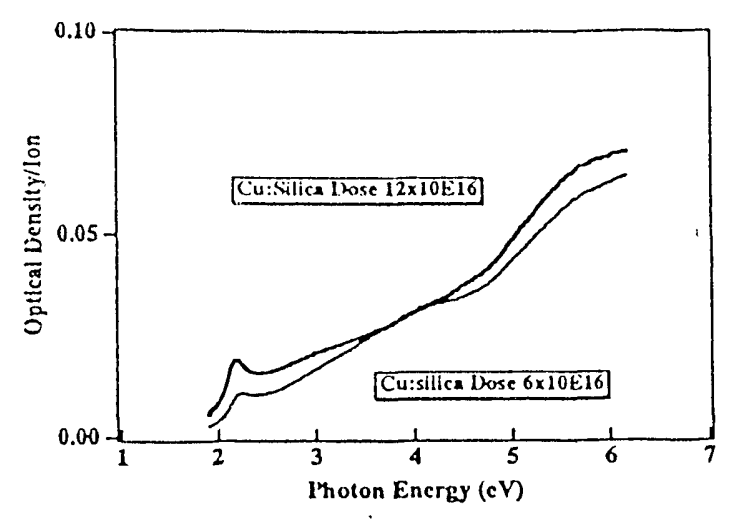

Figure 5. Optical absorption curve, in units of optical density per implanted ion, for the two samples analyzed by TEM from Figure 1 and Figure 2.

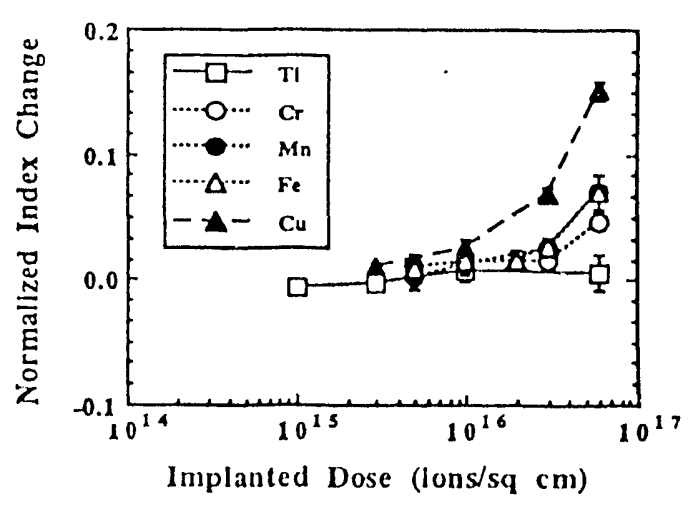

Figure 6. Index of refraction of Cu:silica layers as a function of implarted ion dose, measured by ellipsometry at a wavelength of $633 \mathrm{~nm}$, near the surface plasmon resonance for $\mathrm{Cu}$ clusters.

We have measured the nonlinear refractive index of our samples in our first attempts to assess the potential of optically bistable materials of this type in nonlinear optical devices. The nonlinear index $\gamma$ is defined in terms of its relation to the ordinary linear index $\mathrm{n}_{0}$ and the (complex) third-order nonlinear dielectric susceptibility $\chi^{(3)}$ as follows:

$$
\mathrm{n}=\mathrm{n}_{\mathrm{o}}+\gamma \cdot \mathrm{I}, \quad \gamma=\frac{4 \pi}{3 \mathrm{n}_{\mathrm{o}}} 10^{-8} \cdot \operatorname{Re}\left[\chi^{(3)}\right]
$$

where $\mathrm{n}$ is the total index of refraction and $\mathrm{I}$ is the laser intensity. The nonlinear index may arise from several mechanisms: electronic excitation, the therno-optic effect, electrostriction, and ionic excitations. The mechanism which operates in any particular experiment will depend on the choice of laser pulse length, pulse repetition frequency, and laser wavelength in relation to the electronic structure and electron-phonon coupling in the material.

We measured the nonlinear index of refraction $\gamma$ of $\mathrm{Cu}$-implanted silica by observing variations in the far-field intensity of laser light transmitted through the sample as a function of laser intensity. ${ }^{6}$ The laser was a continuous-wave, mode-locked, frequency-doubled $\mathrm{Nd}$ :YAG laser producing 100-ps pulses at a wavelength of $532 \mathrm{~nm}$ and a pulse-repetition

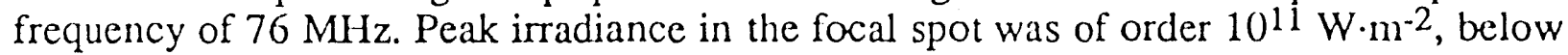
the threshold for thermal damage insofar as that could be observed by visible microscopy. The intensity was varied by translating the sample through the focal plane of a $150-\mathrm{mm}$ focal length lens on a micrometer-driven translation stage. The transmitted intensity was monitored by a power meter located behind a beam-limiting aperture $90 \mathrm{~cm}$ from the focal plane, and normalized to the signal from a beamsplitter located before the focusing lens. Slow thermal drifts were compensated by averaging forward and backward scans. For a material with a positive nonlinear index of refraction, moving the sample toward, and then 
away, from the focal spot causes an initial decrease in the normalized far-field intensity, followed by a crossing through unity at the focal spot and then an increase in intensity. However, the samples became warm to the touch after a few minutes of laser irradiation.

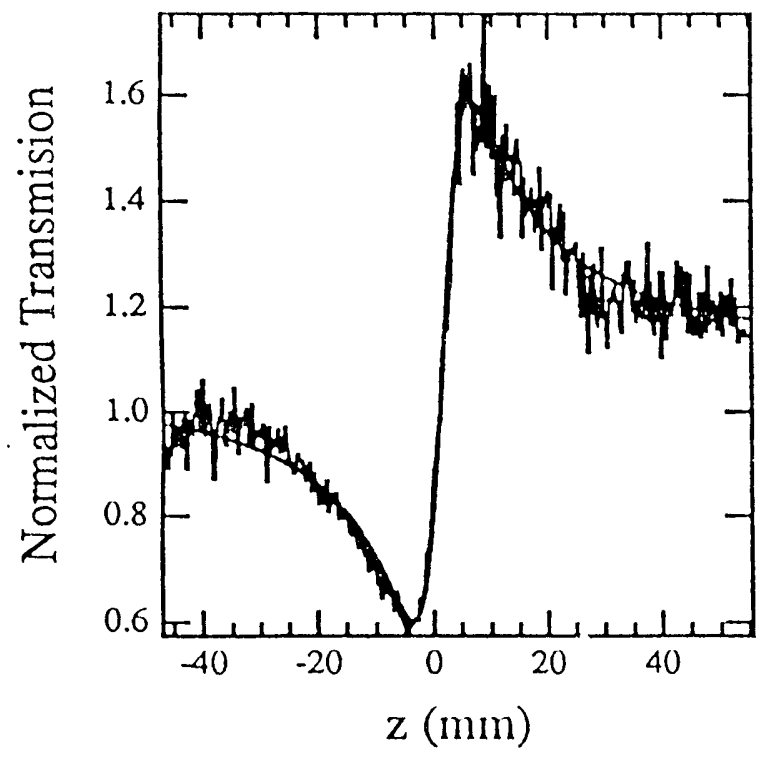

Figure 7. Far-field intensity as a function of sample distance from the focal plane for $\mathrm{Cu}$ :silica $\left(6 \cdot 10^{16}\right.$ ions $\cdot \mathrm{cm}^{-2}$, two sides). The smooth curve drawn through the data is calculated from Eq. (2).

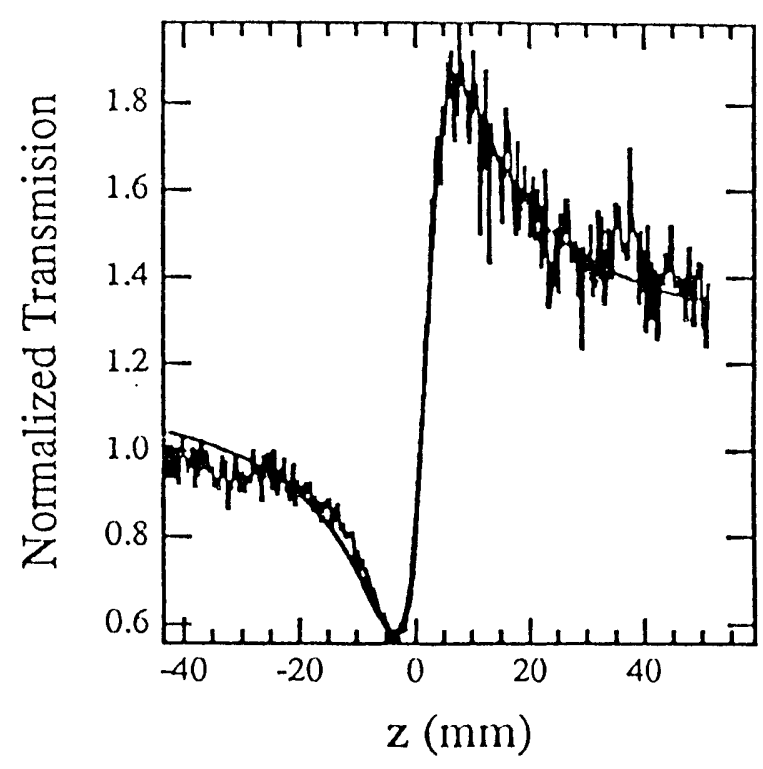

Figure 8. Far field intensity as a function of sample distance from the focal plane for Cu:silica $\left(12 \cdot 10^{16}\right.$ ions $\cdot \mathrm{cm}^{-2}$, one side $)$. The smooth curve drawn through the data is calculated from Eq. (2).

Experimental results for the far-field intensity of laser light transmitted through the two $\mathrm{Cu}$ :silica samples are shown in Figures 7 and 8. For the thin, highly absorbing layers produced by ion implantation, the intensity-dependent change in refractive index $\gamma=\Delta \mathrm{n} / \mathrm{I}$ is extracted from the $\mathrm{Z}$-scan measurement, and for moderate intensities turns out to be

$$
\gamma=\frac{I_{\max }-I_{\min }}{I_{\max }+I_{\min }} \cdot \frac{n_{0} r_{o}^{2}}{z_{o} I_{o} L} \quad, \quad z_{o} \equiv \frac{\pi r_{o}^{2}}{\lambda}
$$

where $I_{\max }$ and $I_{\min }$ are the maximum and minimum intensities recorded in the $\mathrm{Z}$-scan; $\mathrm{I}_{\mathrm{O}}$ is the laser peak intensity at the focal spot, $\mathrm{r}_{\mathrm{O}}$ is the radius of the Gaussian beam profile at the focal plane, $\mathrm{L}$ is the thickness of the implanted layer, and $z_{0}$ is the diffraction length. Note that the difference $\Delta I=I_{\max }-I_{\min }$ for the low-dose sample implanted on both sides (to a dose of $12 \cdot 10^{16}$ ions $\cdot \mathrm{cm}^{-2}$ ) is almost identical to that for the high-dose sample implanted on one side (to a dose of $12 \cdot 10^{16}$ ions $\cdot \mathrm{cm}^{-2}$ ), if one accounts for the Fresnel reflection at the two additional interfaces in the two-side-implanted sample.

We calibrated our results by measuring the nonlinear index of $\mathrm{CS}_{2}$, and obtained the accepted value for its thermo-optic coefficient. By fitting the results of the measurements in the implanted samples to Eq. (2), we find that $\gamma=1.8 \cdot 10^{-7} \mathrm{~cm}^{2} / \mathrm{W}$. Because the extracted values for $\gamma$ are similar for our implanted samples and because the nonlinear index thus seems to be correlated with the total amount of implanted $\mathrm{Cu}$, we conclude that the 
refractive part of the optical nonlinearity measured for these relatively long, high-repetitionrate pulses is probably also thermo-optic in character.

\section{Discussion}

The large values of $\gamma$ measured in these experiments are almost certainly due to the thermooptic effect resulting from the absorption of laser light in the implanted layer, in which the small-signal absorption is of order $10^{4} \mathrm{~cm}^{-1}$. However, noble-metal colloids - in particular, the gold colloids responsible for the beautiful colors of the ruby-gold glasses - are known to have large, ultrafast optical nonlinearities. ${ }^{7}$ Measurements and calculations of the electronic Kerr nonlinearity in Au nanoclusters suggest that the electronic nonlinear susceptibility is dominated by hot-electron absorption, with $\chi^{(3)}$ of order $10^{-7}$.esu. This would correspond to a nonlinear index $\mathrm{n}_{2}=10^{-7} \mathrm{esu}$, or $\gamma=10^{-10} \mathrm{~cm}^{2} \cdot \mathrm{W}^{-1}$, far smaller than the thermo-optic $\gamma$ measured in these particular experiments. Thus, there is every reason to suspect that degenerate four-wave mixing (DFWM) measurements now in progress will reveal an electronic nonlinear susceptibility in these dense nanocluster layers.

\section{Acknowledgements.}

We thank Dr. Klaus Becker of Vanderbilt University for his assistance in the nonlincar optical measurements and analysis. Research at Vanderbilt University was supported in part by the Army Research Office under contract DAAL03-91G-0028. Oak Ridge National Laboratory is partially supported by the Division of Materials Science, U. S. Department of Energy, under contract DE-AC05-84OR21400 with Martin-Marietta Energy Systems, Inc.

\section{References}

1. G. I. Stegemann and R. H. Stolen, "Waveguides and fibers for nonlinear optics," J. Opt. Soc. B 6 (1989) 652-662.

2. K. Becker, Y. Li, R. F. Haglund, Jr., R. H. Magruder III, R. A. Weeks and R. A. Zuhr, "Nonlinear and Fluorescence Properties of Ion-Implanted Fused Silica," Nucl. Instrum. Meth. in Phys. Res. B 59/60 (1991) 1304-1307.

3. R. F. Haglund, Jr., H. C. Mogul, R. A. Weeks and R. A. Zuhr, "Changes in the Refractive index of fused silica due to implantation of transition-metal ions," J. NonCryst. Sol. 130 (1991) 326-331.

4. G. C. Papavassiliou, "Optical Absorption Spectra of Silver, Gold, and Copper Thin Films Chemically Deposited on Quartz Plates," Z. Phys. Chemic (Leipzig) 257 (1976) 241-248.

5. R. F. Haglund, Jr., R. H. Magruder III, D. O. Henderson, S. H. Morgan, L. Yang and R. A. Zuhr, "Nonlinear Index of Refraction in $\mathrm{Cu}$ - and Pb-Implanted Fused Silica," Nucl. Instrum. Meth. in Phys. Res. B, to be published.

6. M. Sheik-Bahae et al., "Sensitive Measurement of Optical Nonlinearities Using a Single Beam," IEEE J. Quantum Elect. 26 (1990) 760-769.

7. F. Hache, D. Ricard, C. Flytzanis and U. Kreibig, "The Optical Kerr Effect in Small Metal Particles and Metal Colloids: The Case of Gold," App. Phys. A 47 (1988) 347-357. 


\section{DISCLAIMER}

This report was prepared as an account of work sponsored by an agency of the United States Government. Neither the United States Government nor any agency thereof, nor any of their employees, makes any warranty, express or implied, or assumes any legal liability or responsibility for the accuracy, completeness, or usefulness of any information, apparatus, product, or process disclosed, or represents that its use would not infringe privately owned rights. Reference herein to any specific commercial product, process, or service by trade name, trademark, manufacturer, or otherwise does not necessarily constitute or imply its endorsement, recommendation, or favoring by the United States Government or any agency thereof. The views and opinions of authors expressed herein do not necessarily state or reflect those of the United States Government or any agency thereof. 

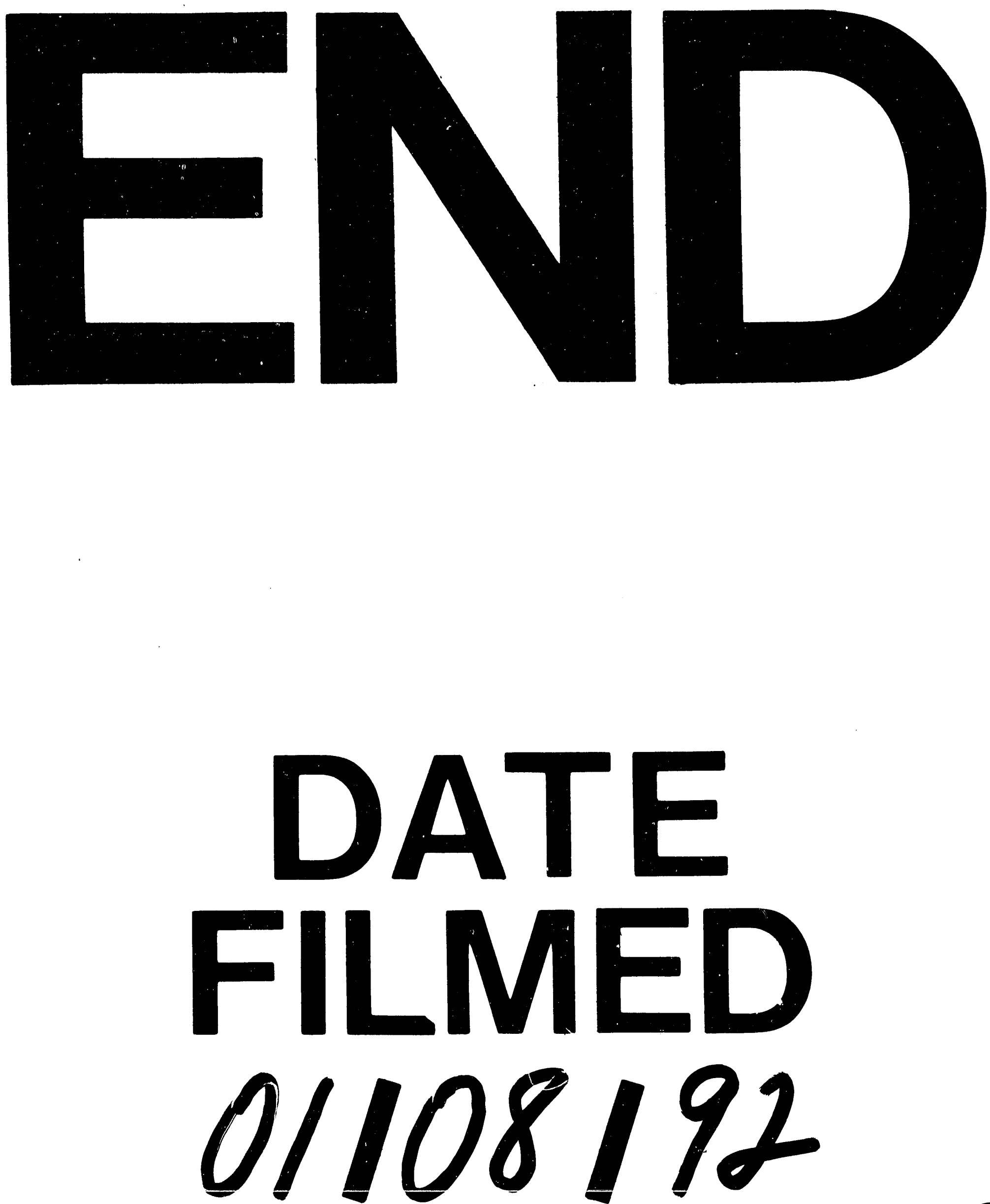
